\title{
ON THE EXISTENCE OF PLANE CURVES WITH PRESCRIBED SINGULARITIES
}

\author{
GERALD B. HUFF
}

1. Introduction. It is the purpose of this lecture to present three problems on the existence of plane curves with prescribed singularities and to give some indication of the present state of these questions. The geometry is the classical algebraic geometry of the plane over the field of complex numbers.

If $n, m, \delta, \kappa$, and $i$ are the order, class, number of double points, number of cusps, and numbers of inflections of an irreducible plane curve $f=0$, these numbers are Pluecker characteristics of $f=0$, and satisfy Pluecker's equations:

$$
\begin{aligned}
m & =n(n-1)-2 \delta-3 \kappa \\
i & =3 n(n-2)-6 \delta-8 \kappa
\end{aligned}
$$

It is natural to raise the question:

I. Given a solution of $(\mathrm{P})$ in non-negative integers, does there exist an irreducible plane curve for which these integers are the Pluecker characteristics?

A solution of $(\mathrm{P})$ with this geometrical interpretation will be said to be proper.

The second question arises in the theory of linear systems of plane curves. A linear system $\Sigma$ is given by

$$
\lambda_{0} f_{0}+\lambda_{1} f_{1}+\cdots+\lambda_{d} f_{d}=0,
$$

where the $\lambda$ 's are parameters and $f_{0}, f_{1}, \cdots, f_{d}$ are ternary forms of order $x_{0}$. If $f_{0}, f_{1}, \cdots, f_{d}$ are linearly independent, then $d$ is the dimension of the linear system $\Sigma$. If $P_{i}$ is a point such that all the curves of $\Sigma$ are on $P_{i}$, then $P_{i}$ is a base point of $\Sigma$. If the general curve of $\Sigma$ has multiplicity $x_{i}$ at $P_{i}$, then $\Sigma$ is said to have multiplicity $x_{i}$ at $P_{i}$. Let $P_{1}, P_{2}, \cdots, P_{p}$ be base points of $\Sigma$ with multiplicities $x_{1}, x_{2}, \cdots, x_{\rho}$. If $\Sigma$ contains all curves of order $x_{0}$ with these multiplicities at these points, then $\Sigma$ is said to be complete (with respect to these multiplicities at these base points). If the linear conditions imposed by asking that a curve of order $x_{0}$ have these multiplicities at these points are independent, then $\Sigma$ is said to be regular (with

An address delivered before the New Orleans Meeting of the Society on April 21, 1951 by invitation of the Committee to Select Hour Speakers for Southeastern Sectional Meetings; received by the editors June 26, 1951. 
respect to these multiplicities at these points). The set of non-negative whole numbers $\left\{x_{0} ; x_{1}, x_{2}, \cdots, x_{\rho}\right\}$ is called the characteristic $x$ of $\Sigma$. If the general curve of the system $\Sigma$ is irreducible and is of genus $p$, then the system is of genus $p$. If $x$ is the characteristic of a complete and regular linear system of dimension $d$ and genus $p$, and for which the general curve is irreducible and has no singularities except those prescribed at the base points, then these integers satisfy Cremona's equations $[5,3]:^{1}$

$$
\begin{aligned}
x_{0}^{2}-x_{1}^{2}-x_{2}^{2}-\cdots-x_{p}^{2} & =d+p-1, \\
3 x_{0}-x_{1}-x_{2}-\cdots-x_{\rho} & =d-p+1 .
\end{aligned}
$$

(If $d>0$, the assumption that the general curve of $\Sigma$ is irreducible implies that $\Sigma$ has no singularities except at the base points.) It is customary to call any set of integers, $x=\left\{x_{0} ; x_{1}, x_{2}, \cdots, x_{\rho}\right\}$, a characteristic, whether or not it arises from a complete and regular linear system. Our second question may be phrased as follows:

II. If $x=\left\{x_{0} ; x_{1}, x_{2}, \cdots, x_{\rho}\right\}$ is a characteristic composed of nonnegative integers which determine non-negative $p$ and $d$ in (C), does there necessarily exist a linear system $\Sigma$ for which $x$ is the characteristic?

A characteristic $x$ which is associated with a linear system in this way is said to be proper (or geometric).

Cremona and his followers were interested in this question in the case $p=0$ and $d=2$. The interest in these linear systems-homaloidal nets-arises from the following fact. If a homaloidal net in a plane $\Pi$ is put into projective correspondence with the (homaloidal) net of lines in a plane $\Pi^{\prime}$, there is induced a point transformation between the planes which is birational and single-valued both ways with the exception of a finite number of points in each plane. Under this Cremona transformation a linear system $\Sigma$ with characteristic $x$ at $\rho$ general points $F_{1}, F_{2}, \cdots, F_{\rho}$ has an image $\Sigma^{\prime}$ with characteristic $x^{\prime}$ at a congruent [1] set of points in $\Pi^{\prime}$. In fact, $x^{\prime}$ is the image of $x$ under a linear substitution with integer coefficients:

$$
\begin{aligned}
& x_{0}^{\prime}=n x_{0}-s_{1} x_{1}-\cdots-s_{\rho} x_{\rho}, \\
& S: \quad x_{1}^{\prime}=r_{1} x_{0}-a_{11} x_{1}-\cdots-a_{1 \rho} x_{\rho}, \\
& \cdots \cdots \cdots \cdots \cdots \cdots \cdots \cdots+a_{\rho \rho} x_{\rho} . \\
& x_{\rho}^{\prime}=r_{\rho} x_{0}-a_{1 \rho} x_{1}-\cdots \cdots
\end{aligned}
$$

Here $\left\{n ; s_{1}, \cdots, s_{\rho}\right\}$ is the characteristic of the homaloidal net which determines the transformation. The integers $n, s_{i}, r_{j}, a_{i j}$ are

${ }^{1}$ Numbers in brackets refer to the references at the end of the paper. 
non-negative and the forms $Q=x_{0}^{2}-x_{1}^{2}-x_{2}^{2}-\cdots-x_{\rho}^{2}$ and $L=3 x_{0}$ $-x_{1}-x_{2}-\cdots-x_{\rho}$ are invariant under $S$. The form of the last question is now clear.

III. If $S$ is a linear substitution of the form above in which the letters are non-negative integers and which leaves $Q$ and $L$ invariant, is this $S$ necessarily associated with a planar Cremona transformation in the way described above?

A linear substitution $S$ which is asociated with a Cremona transformation is said to be geometric.

It is known that the answer to all of these questions is no. For each question, our procedure will be to give a simple example to show that the answer is in the negative and to examine modifications of the question suggested by the example.

2. Improper solutions of Pluecker's equations. In Salmon-Fiedler [18], mention is made of a curve with $n=7, \delta=0$, and $\kappa=13$, which makes $m=3$ and $i=1$ in the equations $(\mathrm{P})$. Recall now that the Pluecker characteristics of a curve also satisfy the dual equations:

$$
\begin{aligned}
& n=m(m-1)-2 t-3 i, \\
& \kappa=3 m(m-2)-6 t-8 i,
\end{aligned}
$$

where $t$ designates the number of bitangents and the remaining letters have the meaning described above. Substituting the numbers above into the first of these dual equations, we see that $\left(\mathrm{P}^{*}\right)$ yields $t=-2$ and we conclude that there is no curve of order 7 with no double points and thirteen cusps.

While it is necessary to require that all the values of $n, m, \delta, \kappa, i, t$ must be non-negative to insure the existence of a curve for which any subset are Pluecker characteristics, it is still easy to construct an example showing that this is also not sufficient. A simple example is $n=m=4, \delta=t=4$, and $\kappa=i=0$. When an irreducible curve does exist, its genus $p$ is given by

$$
p=(n-1)(n-2) / 2-\delta-\kappa .
$$

Thus we are led to a modification of the original equation which has received considerable attention.

$\mathrm{I}_{2}$. Given a solution of $(\mathrm{P})$ and $\left(\mathrm{P}^{*}\right)$ in non-negative integers such that $p=(n-1)(n-2) / 2-\delta-\kappa$ is also non-negative, does there exist an irreducible plane curve for which these integers are the Pluecker characteristics?

In his well-known article on the principle of projection and section, Veronese [20] showed that the answer to $I_{a}$ is yes for $p=0$. Later, 
Lefschetz, in a much quoted paper [15], showed that the answer to $I_{a}$ is in the affirmative for $p=0,1$, and 2 . He further showed that the same would be true for $p=3$ if it were possible to construct a curve for $n=7, \delta=1$, and $\kappa=11$. In 1931 , while studying problems about algebraic surfaces [21], Zariski obtained a curious theorem about the cusps of a plane curve. Let a plane curve of order $n$ have cusps and let $j$ be an integer such that $6 j<n$. The theorem asserts that the linear system of curves of order $n-3-j$ with simple points at the cusps is a regular linear system. Now suppose that a curve of order 7 with 11 cusps can exist. It then follows that there exists a regular linear system of cubics on eleven points and this makes the dimension -2 ! This theorem thus shows that even the modified question $I_{a}$ must also be answered in the negative.

Lefschetz and Zariski made use of the fact that if $n, p, \kappa$ are given, then the remaining characteristics are determined as integers. They studied the arithmetic conditions imposed on $n, p$, and $\kappa$ by the fact that these remaining integers must not be negative in a proper solution. Finally, Zariski [22] gave a set of inequalities satisfied by $n, p$, and $\kappa$ which were necessary and sufficient conditions that the remaining integers be non-negative. To these he adjoined one more inequality which insures that the solution corresponding to $n, p, \kappa$ cannot be shown to be improper by his theorem. The final form of the first question may be put as follows:

$\mathrm{I}_{\mathrm{z}}$. Is there an irreducible plane curve corresponding to every $n, p, \kappa$ which satisfies the Zariski inequalities?

This is at present an open question.

3. Improper solutions of Cremona's equations. Even in the classical case, $p=0, d=2$ studied by Cremona and his followers $[5 ; 12 ; 14]$, there are non-negative solutions of equations $(C)$ which are not associated with homaloidal nets. It is customary here to refer to the fact that Ruffini [17] erroneously gave $\left\{10 ; 6,4,3^{5}, 1^{2}\right\}$ as the characteristic of a homaloidal net. For our purposes we shall consider the simplest nongeometric solution,

$$
\text { (0) } x=\{5 ; 3,3,1,1,1,1,1,1\} \text {. }
$$

To see that no homaloidal net corresponds to this solution we may reason as follows. If there were a homaloidal net of this description, it would determine a linear substitution $S$ with $\{5 ; 3,3,1,1,1,1,1,1\}$ in the first row and with $Q, L$ as invariants. Now one may make a diophantine argument to show that the remaining coefficients can not all be integers. This shows that $\{5 ; 3,3,1,1,1,1,1,1\}$ is non- 
geometric and suggests also the following modification of question II for $p=0, d=2$.

$\mathrm{II}_{\mathrm{a}}$. Is there a homaloidal net associated with every solution of (C) for $p=0, d=2$ which appears in the first row of a linear subsitution $S$ which has $Q, L$ as invariants? This conjecture has been repeated in the literature $[1 ; 2]$ and is easily seen to be equivalent to the original question III. Although one may find incomplete arguments in the literature $[4 ; 13]$ which claim to show that the answer is in the affirmative, we shall see in a moment that it is easy to construct an example showing that the answer is no.

It should first be pointed out that there is a simpler way of showing that $\{5 ; 3,3,1,1,1,1,1,1\}$ is not a geometric solution of equations (C). If there were indeed a linear system of quintics with an irreducible general curve, then the line through the two triple base points would meet this curve in at least six points and this asserts that the curve is reducible. This shows completely that the example is not geometric and suggests a further necessary condition for proper solution. If $x$ is proper and $c$ is the characteristic of a curve of $c_{0}<x_{0}$, then

$$
c_{0} x_{0}-c_{1} x_{1}-c_{2} x_{2}-\cdots-c_{\rho} x_{\rho} \geqq 0 .
$$

In an hour address to the society over a quarter of a century ago, Coble [2] suggested that any solution of (C) for $p=0, d=2$ which also satisfied all possible inequalities of the form (L) must then be proper. Here we shall modify our second question to:

$\mathrm{II}_{\mathrm{b}}$. Is there a linear system with irreducible general curve associated with every solution of equations $(\mathrm{C})$ which also satisfy inequalities $(\mathrm{L})$ ?

4. The invariant theory of Cremona's equations. There does exist a reasonably simple theory of characteristics which permits a systematic attack on these problems. If, for a fixed $\rho, S$ and $S^{*}$ are two integer substitutions which are geometric, then it may be shown that $S S^{*}$ is also geometric. This is the decisive step in showing that the collection of all geometric $S$ for a given $\rho$ form a group, which we shall designate by $G_{\rho}$.

Associated with a quadratic transformation there is the substitution:

$$
A_{123}: \quad x_{i}^{\prime}=x_{i}+a_{i}\left(a_{0} x_{0}-a_{1} x_{1}-\cdots-a_{\rho} x_{\rho}\right), \quad i=0,1, \cdots, \rho,
$$

where $a$ is the vector $\{1 ; 1,1,1,0, \cdots, 0\}$. For any $\rho \geqq 3, A_{123} \in G_{\rho}$. It is further true that any substitution which represents a permutation of $x_{1}, x_{2}, \cdots, x_{\rho}$ is in $G_{\rho}$ [2]. We shall designate the group gen- 
erated by $A_{123}$ and the permutations by ( $A_{123}$, permutations). It is certainly a subgroup of $G_{\rho}$.

If $T$ is a linear substitution which leaves $Q$ unchanged, then it follows-exactly as for orthogonal substitutions-that the determinant of $T$ is \pm 1 . Thus the set of all linear substitutions with integer coefficients and with $Q$ and $L$ as invariants must be a group, which we shall call $G_{\rho}(I)$. The set of those elements of $G_{\rho}(I)$ which have $n, s_{i}, r_{j}, a_{i j}$ non-negative in the form $(S)$ form a complex of $G_{\rho}(I)$ which will be designated by $H_{\rho}\left(I^{+}\right)$. It is clear from these definitions that

$$
\text { ( } A_{123}, \text { permutations) } \subseteq G_{\rho} \subseteq H_{\rho}\left(I^{+}\right) \subseteq G_{\rho}(I) .
$$

Several of the significant results in this connection can now be stated in terms of whether or not these inclusions are proper inclusions.

For $\rho \leqq 8$, these sets are all finite and are all the same. Du Val has studied the groups in detail [6] and has identified them with certain crystallographic groups. For $\rho=9$, the sets are all infinite, but even here, all these sets are the same [19]. Notice that our original question III, in this notation, becomes: Is $G_{\rho}=H_{\rho}\left(I^{+}\right)$? For $\rho \leqq 9$, questions III and $\mathrm{II}_{\mathrm{a}}$ must be answered in the affirmative.

For $\rho>9$, the situation is different. We should first mention that in a celebrated paper [16] M. Noether showed that

(1) For all $\rho,\left(A_{123}\right.$, permutations $)=G_{\rho}$. That is, $G_{\rho}$ is generated by $A_{123}$ and the permutations of $x_{1}, x_{2}, \cdots, x_{\rho}$.

(Actually, Noether announced a more general result at that time, although this is exactly what his argument demonstrated.) In 1934 Coble constructed an example, for $\rho=10$, of an element of $G_{\rho}(I)$ not in $H_{\rho}\left(I^{+}\right)$[3]:

(2) For $\left.\rho \geqq 10, H_{\rho}\left(I^{+}\right)\right) \subset G_{\rho}(I)$.

Much earlier, S. Kantor, in his prize memoir crowned by the Naples Academy $[13 ; 4]$, had claimed that $G_{\rho}=H_{\rho}\left(I^{+}\right)$and had supported the assertion by an argument containing an incomplete induction. If we pause here to see how Coble constructed his example to prove (2), it will be clear that the faulty argument gave an incorrect answer. Coble thought of $Q=0$ and $L=0$ as quadric and hyperplane in a projective space of $\rho$ dimensions and reasoned that the harmonic perspectivity in $L=0$ and its pole would leave these invariant. He found that these could be written with integer coefficients for $\rho=7,8,10$, and 11. The substitution for $\rho=10$ was Coble's example. By similar geometric reasoning it may be shown that if $a=\left\{a_{0} ; a_{1}, \cdots, a_{\rho}\right\}$ is an integer point in $L=0$, and such that 
$a_{0}^{2}-a_{1}^{2}-\cdots-a_{\rho}^{2}=-2$, then the linear substitution of the form of $A_{123}$ for this $a$ has $Q$ and $L$ as invariants. Three more ones adjoined to the counter example (0) gives $a=\{5 ; 3,3,1,1,1,1,1,1,1,1,1\}$ as an $a$ satisfying these conditions and an element of $H_{11}\left(I^{+}\right)$, which is obviously not in $G_{11}$ [7]:

(3) For $\rho \geqq 11, G_{\rho} \subset H_{\rho}\left(I^{+}\right)$, and the answer to question III is in the negative.

A study of the invariants of $G_{\rho}$ is the setting in which we shall consider the remaining question $\mathrm{II}_{\mathrm{b}}$. It is an almost direct consequence of the definition that:

(4) If $x$ is a proper characteristic and $g \in G_{\rho}$, then $x^{\prime}=g x$ is also proper. Properness is an invariant of $G_{\rho}$.

This is true only if one adopts the rather curious convention that $\{0 ;-1,0, \cdots, 0\}$ is a proper characteristic for $p=d=0$, and is a consequence of the fact that this is the image of the proper characteristic $\{1 ; 0,1,1,0, \cdots, 0\}$ under $A_{123}$. Even with this exceptional proper characteristic, $x_{0}, p$, and $d$ are non-negative for all proper characteristics. Let $A_{\rho}$ be the set of all characteristics such that $x_{0}, p$, and $d$ are non-negative. Then by using the fact (1) that the generators of $G_{\rho}$ are known, it may be shown that [8]:

(5) If $x \in A_{\rho}$ and $g \in G_{\rho}$, then $x^{\prime}=g x$ is in $A_{\rho}$. The property of being in $A_{\rho}$ is an invariant of $G_{\rho}$.

It is not known if the inequalities (L) are invariant under $G_{\rho}$, but the following is a result of this sort. Let $E_{\rho}$ be the set of proper solutions of (C) for $p=d=0$. Then if $x$ is a proper solution of (C), $e \in E_{\rho}$ and $e_{0}<x_{0}$, it follows as before that:

$$
e_{0} x_{0}-e_{1} x_{1}-\cdots-e_{\rho} x_{\rho} \geqq 0, \quad e \in E_{\rho} \text { and } e_{0}<x_{0} .
$$

These are called the properness inequalities. It may be shown that [9]:

(6) If $x \in A$ satisfies (PI) and $g \in G_{\rho}$, then $x^{\prime}=g x$ also satisfies (PI). Satisfaction of the properness inequalities is an invariant of $G_{\rho}$.

Now let $x \in A$ and let $G_{\rho}(x)$ be the set of images of $x$ under $G_{\rho}$. In accordance with (5), if $y \in G_{\rho}(x)$, then $y_{0} \geqq 0$, and there is an element $x^{*}$ in $G(x)$ such that $x^{*}$ is of minimal order; that is, $x_{0}^{*}$ is a minimum. The result we need here is that [10]

(7) A characteristic $x$ is of minimal order if and only if $x_{0}-x_{i}-x_{j}$ $-x_{k} \geqq 0$, where $x_{i}, x_{j}, x_{k}$ are three of the largest among $x_{1}, \cdots, x_{\rho}$.

We may now ask if all solutions of (C) and (PI) are indeed proper. If $x$ is a solution of (C) and (PI), then $G_{\rho}(x)$ contains a minimal order element $x^{*}$ satisfying (C) and (PI) and the condition of (7). Since properness is an invariant, $x$ is proper if and only if $x^{*}$ is. But it is possible to tabulate all solutions of (C), (PI), and (7) for $p=0$ and 
1. This leads to the conclusion [10]:

(8) For $p=0$ and any $d$, all solutions of (C) and (PI) are proper. For $p=1$ and $d$ positive, all solutions of $(\mathrm{C})$ and (PI) are proper. For $p=1$ and $d=0$, any solution of $(\mathrm{C})$ and $(\mathrm{PI})$ of g.c.d. $=1$ is proper.

Since for any $x$, the inequalities (PI) are included among the inequalities (L), this means that Coble's conjecture is settled: any characteristic $x$ which gives $p=0$ and $d=2$ in (C) and also satisfies the inequalities (L) must be proper. It also gives the answer to the more general question $\mathrm{II}_{\mathrm{b}}$ for $p=0$ and 1 . For arbitrary values of $p$, $\mathrm{II}_{b}$ remains an open question.

On the other hand, the problem of giving an arithmetic description of geometric linear substitutions may be regarded as settled. The result (5) states that a geometric substitution $S$ must send elements of $A_{\rho}$ into elements of $A_{\rho}$. It may be shown that (8) implies that this is also sufficient [11]:

(9) A linear substitution with integer coefficients which leaves $Q$ and $L$ invariant and sends elements of $A_{\rho}$ into elements of $A_{\rho}$ must be geometric.

It should be stated at this point that in restricting our attention to regular linear systems, we have neglected substantial results of J. A. Todd, Nollet, and Jongmans on the existence of linear systems with base points in special position. We close by listing several problems suggested by the ideas we have considered. Is there an appropriate group associated with Pluecker's equations which will permit arguments like those made for Cremona's equations? Are there results like (8) for any values of $p$ and $d$ ? What is the most general abstract setting in which these methods have application?

\section{REFERENCES}

1. A. B. Coble, Algebraic geometry and theta functions, Amer. Math. Soc. Colloquium Publications, vol. 10, 1929, pp. 10, 12.

2. ㄴ. Cremona transformations . . ., Bull. Amer. Math. Soc. vol. 28 (1922) p. 335.

3. - Cremona's diophantine equations, Amer. J. Math. vol. 56 (1934) p. 487.

4. J. L. Coolidge, Algebraic plane curves, Oxford, 1931, p. 487.

5. L. Cremona, Sulle transformazioni geometriche delle figure piane, Giornale di Matematiche vol. 1 (1863) p. 306.

-6. P. Du Val, On the Kantor group of a set of points in the plane, Proc. London Math. Soc. (2) vol. 42 (1936) pp. 18-51.

7. G. B. Huff, $A$ note on Cremona transformations, Proc. Nat. Acad. Sci. U.S.A. vol. 20 (1934) p. 429.

8. - Inequalities connecting solutions of Cremona's equations, Bull. Amer. Math. Soc. vol. 52 (1946) p. 289.

9. - An arithmetic characterization of proper characteristics, Amer. J. Math. vol. 48 (1946) p. 686. 
10. - Cremona's equations and the properness inequalities, Duke Math. J. vol. 17 (1950) p. 388.

11. - The completion of a theorem of Kantor, Bull. Amer. Math. Soc. vol. 50 (1944) p. 694.

12. E. de Jonquieres, Solution d'une question d'analyse indeterminé, C. R. Acad. Sci. Paris vol. 101 (1885) p. 857.

13. S. Kantor, Premier fondements pour une theorie des transformations univoques..., Naples, 1891, p. 297.

14. I. Larice, Due nuove soluzioni generali ... delle equazioni di condizione della trasformazioni Cremoniani, Periodico di Matematica per l'Insegnamento Secondario (3) vol. 6, p. 234.

15. S. Lefschetz, On the existence of loci with given singularities, Trans. Amer. Math. Soc. vol. 14 (1913) p. 23.

16. M. Noether, Ueber Flächen, welche Schaaren rationaler Kurven besitzen, Math. Ann. vol. 3 (1871) p. 166.

17. F. P. Ruffini, Risoluzione di 2 equazione ... di trasformazione Cremoniana, Memorie della R. Accademia delle scienze dell' Istituto di Bologna vol. 8 (1877) p. 483.

18. G. Salmon and O. W. Fiedler, Analytische Geometrie der höheren ebenen Kurven, Leipzig, 1882, p. 83.

19. M. E. Taylor, $A$ determination of the types of Cremona transformations, Amer. J. Math. vol. 54 (1932) p. 123.

20. G. Veronese,.... das Princip des Projicirens und Schneidens .... Math. Ann. vol. 19 (1882) p. 211.

21. O. Zariski, On the irregularity of cyclic multiple planes, Ann. of Math. vol. 32 (1931) p. 485.

22. - Algebraic surfaces, Springer, 1935, p. 171.

The University of Georgia 